\title{
Cell type-specific deletion in mice reveals roles for PAS kinase in insulin and glucagon production
}

\author{
Francesca Semplici $^{1}$ • Angeles Mondragon ${ }^{1} \cdot$ Benedict $_{\text {Macintyre }}{ }^{1} \cdot$ \\ Katja Madeyski-Bengston ${ }^{2,3}$ - Anette Persson-Kry ${ }^{2,3} \cdot$ Sara Barr $^{2,3}$ - Anna Ramne R $^{2,3}$ \\ Anna Marley ${ }^{4}$ - James McGinty ${ }^{5} \cdot$ Paul French $^{5}$ • Helen Soedling ${ }^{1}$. \\ Ryohsuke Yokosuka $^{1}$ - Julien Gaitan ${ }^{6}$ • Jochen Lang ${ }^{6} \cdot$ Stephanie Migrenne-Li $^{7}$ • \\ Erwann Philippe $^{7}$ • Pedro L. Herrera ${ }^{8}$ - Christophe Magnan ${ }^{7}$. \\ Gabriela da Silva Xavier ${ }^{1}$ - Guy A. Rutter ${ }^{1}$
}

Received: 11 March 2016 / Accepted: 1 June 2016 / Published online: 24 June 2016

(C) The Author(s) 2016. This article is published with open access at Springerlink.com

\begin{abstract}
Aims/hypothesis Per-Arnt-Sim kinase (PASK) is a nutrientregulated domain-containing protein kinase previously implicated in the control of insulin gene expression and glucagon secretion. Here, we explore the roles of PASK in the control of islet hormone release, by generating mice with selective deletion of the Pask gene in pancreatic beta or alpha cells.

Methods Floxed alleles of Pask were produced by homologous recombination and animals bred with mice bearing beta (Ins $1^{\mathrm{Cre}} ;$ PaskBKO) or alpha $\left(\mathrm{Ppg}^{\mathrm{Cre}}\right.$ [also known as $\mathrm{Gcg}$ ]; PaskAKO) cell-selective Cre recombinase alleles. Glucose homeostasis and hormone secretion in vivo and in vitro, gene expression and islet cell mass were measured using standard techniques.

Results Ins $1^{\mathrm{Cre}}$-based recombination led to efficient beta celltargeted deletion of Pask. Beta cell mass was reduced by
\end{abstract}

Francesca Semplici and Gabriela da Silva Xavier contributed equally to this study.

Electronic supplementary material The online version of this article (doi:10.1007/s00125-016-4025-1) contains peer-reviewed but unedited supplementary material, which is available to authorised users.

Gabriela da Silva Xavier

g.dasilva-xavier@imperial.ac.uk

$\triangle$ Guy A. Rutter

g.rutter@imperial.ac.uk

1 Section of Cell Biology and Functional Genomics, Division of Diabetes, Endocrinology and Metabolism, Department of Medicine, Imperial College London, Imperial Centre for Translational and Experimental Medicine, Hammersmith Hospital, du Cane Road, London W12 0NN, UK

2 AstraZeneca R\&D, DECS, AstraZeneca R\&D, Mölndal, Sweden

$36.5 \%(p<0.05)$ compared with controls in PaskBKO mice, as well as in global Pask-null mice (38\%, $p<0.05)$. PaskBKO mice displayed normal body weight and fasting glycaemia, but slightly impaired glucose tolerance, and beta cell proliferation, after maintenance on a high-fat diet. Whilst glucose tolerance was unaffected in PaskAKO mice, glucose infusion rates were increased, and glucagon secretion tended to be lower, during hypoglycaemic clamps. Although alpha cell mass was increased $(21.9 \%, p<0.05)$, glucagon release at low glucose was impaired $(p<0.05)$ in PaskAKO islets.

Conclusions/interpretation The findings demonstrate cellautonomous roles for PASK in the control of pancreatic endocrine hormone secretion. Differences between the glycaemic phenotype of global vs cell type-specific null mice suggest important roles for tissue interactions in the control of glycaemia by PASK.

4 AstraZeneca R\&D, Alderley Edge, UK

5 Photonics Group, Department of Physics, Imperial College London, London, UK

6 Université de Bordeaux, Institut de Chimie et Biologie des Membranes et des Nano-objets, CNRS UMR 5248, Pessac, France

7 Paris Diderot University, Unit of Functional and Adaptive Biology (BFA), CNRS UMR 8251, Paris, France

8 Department of Genetic Medicine and Development, Faculty of Medicine, University of Geneva, Geneva, Switzerland 
Keywords Basic science $\cdot$ Beta cell signal transduction . Islets $\cdot$ Mouse $\cdot$ PAS kinase

\begin{tabular}{|c|c|}
\hline \multicolumn{2}{|c|}{ Abbreviations } \\
\hline PAS & Per-Arnt-Sim \\
\hline PASK & Per-Arnt-Sim kinase \\
\hline PaskAKO & Alpha cell-selective deletion of Pask \\
\hline PaskBKO & Beta cell-selective deletion of Pask \\
\hline qPCR & Quantitative real-time PCR \\
\hline WT & Wild-type \\
\hline
\end{tabular}

\section{Introduction}

Type 2 diabetes affects approximately one in 12 of the adult population [1] and usually involves changes in both insulin secretion [2] and insulin action [3]. Acting on the healthy pancreatic beta cell, elevated blood glucose concentrations lead to increased glucose flux across the plasma membrane, mediated by the transporter GLUT2 (plus GLUT1 in humans) [4], glucose phosphorylation catalysed by glucokinase, and enhanced glycolytic flux to fuel the citrate cycle [5]. Increased mitochondrial metabolism [6], ATP synthesis [7] and the closure of ATP-sensitive $\mathrm{K}^{+}$channels [8] cause plasma membrane depolarisation, $\mathrm{Ca}^{2+}$ influx via voltage-gated channels (L-type) [9], the further activation of mitochondrial metabolism [10], and secretory granule exocytosis [11]. In addition, ATP-sensitive $\mathrm{K}^{+}$channel-independent mechanisms potentiate the actions of $\mathrm{Ca}^{2+}$ via mechanisms that are presently poorly understood [12]. The signalling pathways controlling glucagon secretion are less well defined and may involve a more dominant role for neural and hormonal control [13], as well as the cell-intrinsic actions of glucose. Enhanced ATP synthesis [14], ATP-sensitive $\mathrm{K}^{+}$channel closure and the opening of T-type $\mathrm{Ca}^{2+}$ channels [15], and ATP-dependent effects on intracellular $\mathrm{Ca}^{2+}$ stores may also be involved [16].

Fuel-sensing kinases such as AMP-activated protein kinase [17] have previously been implicated in the control of both insulin [18, 19] and glucagon secretion [20, 21]. Originally cloned in 2001 by Hofer and colleagues [22] and, separately, by Rutter and colleagues [23], Per-Arnt-Sim (PAS) domaincontaining protein kinase (PASK, also known as PASKIN) is a member of the nutrient-regulated protein-serine kinase family and the only PAS domain-containing member of this group expressed in mammalian cells [24]. In Saccharomyces cerevisiae there are two PASK orthologues: Psk1 and Psk2. Under cell wall stress conditions these enzymes phosphorylate the metabolic enzyme uridine diphosphate-glucose pyrophosphorylase to mobilise glucose [25]. Importantly, the PAS domain of the mammalian enzyme is potentially targetable by small molecules and as such may offer an attractive target for therapeutic intervention in some forms of diabetes [24].

Implying a potentially important role for PASK in regulated insulin secretion, we showed in 2004 that PASK is required in clonal pancreatic beta cells for normal proinsulin gene expression [26]. Subsequently, Rutter and colleagues [27] demonstrated a role for PASK in mammalian glucose homeostasis by showing that mice deleted globally for the enzyme displayed improved insulin sensitivity but impaired insulin secretion in vivo and from isolated islets. We have also shown that activating mutations in the human PASK gene modulate insulin secretion [28]. However, subsequent studies [29] revealed little effect of global Pask deletion in mice on in vivo insulin secretion, but a profound effect on total pancreatic insulin content, consistent with a role for PASK in insulin gene expression $[26,29,30]$ and insulin protein stability [31]. Glucagon release was enhanced in this model [29].

The impact of tissue-selective deletion of conditional Pask alleles has not hitherto been explored, restricting our understanding of the role of the kinase in glucose homeostasis in vivo. Here, we describe the generation of floxed alleles of the kinase and explore the impact of its deletion from pancreatic beta or alpha cells. Whereas fasting and fed insulin levels and glucose-stimulated insulin secretion were not affected by Pask deletion under most conditions, beta cell mass was significantly (36.5\%) reduced in beta cell-selective null animals (PaskBKO). On the other hand, alpha cell-selective deletion (PaskAKO) led to impaired glucagon secretion in vitro and in vivo. These findings demonstrate the importance of PASK for the normal development and function of the beta and alpha cell, respectively.

\section{Methods}

Generation of PaskBKO and PaskAKO mice Mice carrying conditional null alleles of Pask $\left(\right.$ Pask $\left.^{f l f f l}\right)$ were generated

Fig. 1 Generation of conditional Pask alleles and deletion in beta or alpha cells. (a) Knockout (KO) strategy. Generation of beta (PaskBKO) and alpha (PaskAKO) cell-specific Pask KO mice by Cre-mediated excision of exons from 12 to 15 encoding the PASK Ser/Thr kinase domain. Human PASK cDNA (modified from [22]) with the Ser/Thr kinase domain encoding exons circled in purple and enlarged in the black box - a view from the back of PASK kinase domain (amino acids 977-1300) crystal structure (PBD code 3DLS) with the bound ADP molecule in evidence [43]. (b) Mouse Pask gene structure (modified from [22]) and the location of the loxP sites. (c) Example of a genotyping gel indicating the presence of WT and recombined (Cond) alleles. (d) Pask and Ins 2 gene expression measured by qPCR in PaskBKO (BKO) and control (WT) mouse islets. Immunohistochemical analysis of pancreatic sections from PaskBKO (e) and PaskAKO (f) mice and controls, with PASK revealed using Alexa 568 (red), and insulin or glucagon revealed using Alexa 488 (green). Insets $\mathrm{i}$-iv are magnified areas, as shown in the main images. Insets $\mathrm{v}$-viii are example images of no-primary-antibody controls for each of the genotypes, with insets $\mathrm{v}$ and vii showing the DAPI staining for nuclei; scale bars, $100 \mu \mathrm{m}$. PASK fluorescence was displayed in $87.6 \pm 1.5 \%$ of insulinpositive cells in WT islets vs $10.5 \pm 2.3 \%$ in PaskBKO islets; $n=7$ mice per genotype for both PaskBKO and PaskAKO mice; ${ }^{*} p<0.05$ and ${ }^{* *} p<0.01$ by Student's $t$ test. Data were obtained by counting pixel overlay from ten pancreatic slices (typically counting approximately ten islets per slice) per mouse using Fiji (see ESM Methods) 
a

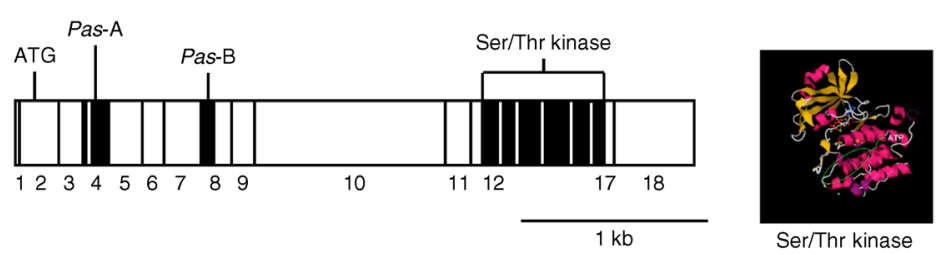

b

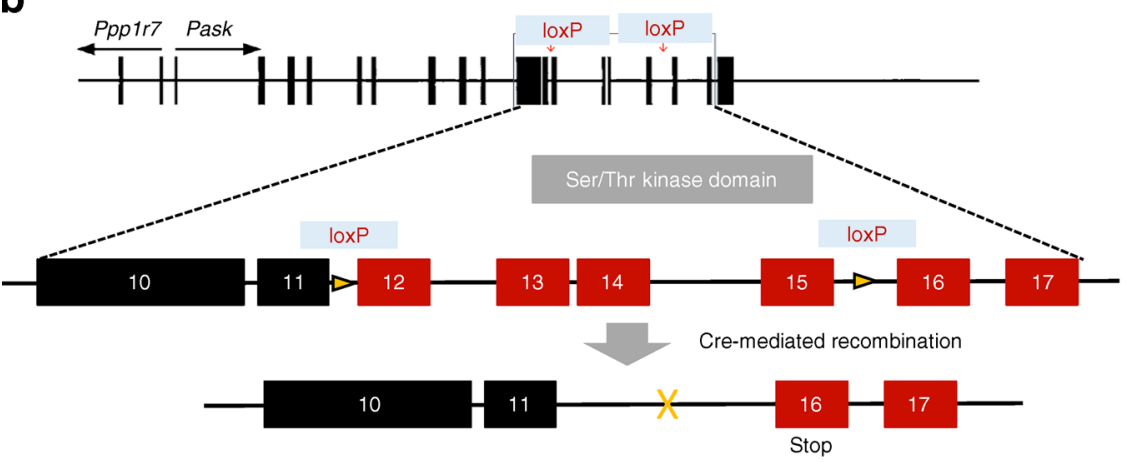

C
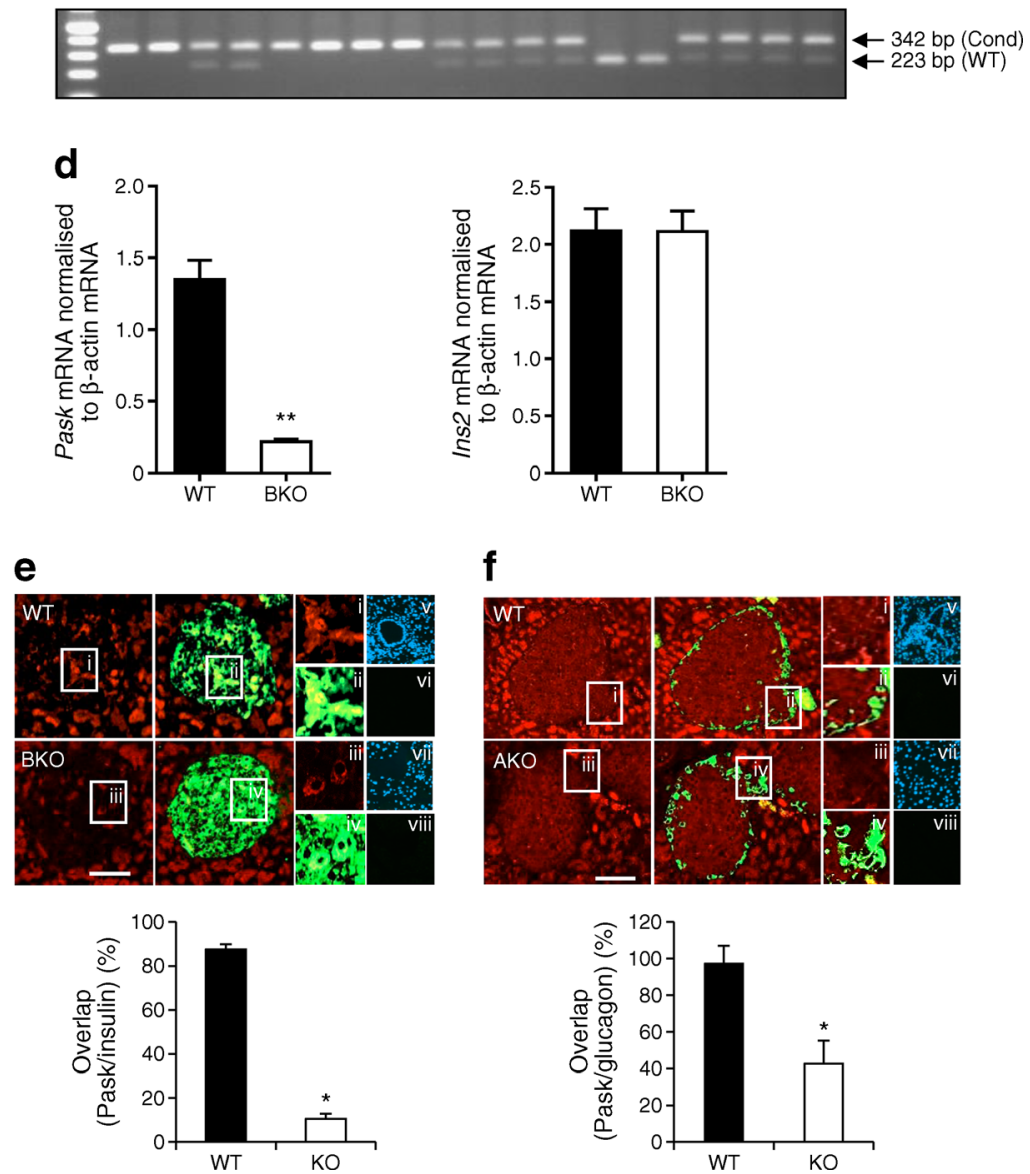

by insertion of loxP sites flanking exon 11 and exon 15 , which encompass the Ser/Thr kinase domain, through homologous recombination (see electronic supplementary material [ESM] Methods, ESM Fig. 1a-c).

Pask $^{f l / f l}$ mice were crossed with animals expressing Cre recombinase under the control of the insulin 1 promoter (Ins ${ }^{\mathrm{Cre}}$ mice [32]) or with mice expressing Cre under the control of the glucagon promoter $\left(\mathrm{Ppg}^{\mathrm{Cre}}\right.$ 
mice [33]) to achieve deletion in pancreatic beta cells (PaskBKO) or in pancreatic alpha cells (PaskAKO). PaskBKO mice were born at the expected Mendelian ratios. PaskAKO mice were born at the expected Mendelian ratios only if assuming that the Cre transgene had co-localised on the same chromosome (chromosome 1) as the Pask floxed gene. Genotyping was performed by PCR using DNA from ear biopsies. Ablation of Pask gene expression from pancreatic islets was assessed by quantitative real-time PCR (qPCR) on cDNA reverse-transcribed from islet RNA, as described below. All mouse strains were maintained on a C57BL/ 6 background. Mice with global deletion of Pask [34] were gifts from R. Wenger (University of Zürich, Zürich, Switzerland).

Mouse maintenance and diet Animals were housed in groups of two to five per individually ventilated cage in a pathogen-free facility with a $12 \mathrm{~h}$ light/dark cycle and were fed ad libitum with a standard mouse chow diet or a high-fat diet $(60 \% \mathrm{wt} / \mathrm{wt}$ fat content; Research Diets, New Brunswick, NJ, USA). Where indicated, 8-week-old mice were transferred on to a high-fat diet for a period of 12 weeks. All in vivo procedures described were performed at the Imperial College Central Biomedical Service and approved by the UK Home Office according to the UK Animals (Scientific Procedures) Act 1986 (PPL 70/7349 and PPL 70/7179).

In vivo physiology (IPGTT, in vivo glucosestimulated insulin secretion, insulin tolerance tests, plasma glucagon, hyperinsulinaemic-hypoglycaemic clamps), RNA extraction and qPCR, islet isolation and hormone secretion, and beta and alpha cell mass measurements are described in ESM Methods. Blood was collected at indicated time points and plasma insulin was measured by ELISA (Mercodia, Uppsala, Sweden). Total cellular RNA was extracted from mouse islets or other tissues and converted to cDNA for qPCR. Alpha and beta cell mass was assessed in pancreases from 20-week-old mice. Anti-PASK antibody (PA5-29309; Pierce, Rockford, IL, USA) was used in immunohistochemical analysis. Experimenters were blinded to the group assignment for assessment of islet cell mass. Samples were not randomised. No data, samples or animals were excluded.

Statistical analysis Data are expressed as means \pm SEM. Significance was tested by unpaired or paired twosample Student's $t$ tests using Excel, or by ANOVA using GraphPad 4.0 (La Jolla, CA, USA). A value of $p<0.05$ was considered significant.

\section{Results}

Generation of mice deleted for PASK selectively in the pancreatic beta or alpha cell Breeding of mice with floxed Pask alleles with animals expressing Cre recombinase under the control of the Ins 1 [32] or Ppg gene promoter [33] was predicted to lead to recombination selectively in pancreatic islet beta (PaskBKO) or alpha (PaskAKO) cells, respectively. Correspondingly, Pask mRNA levels, assessed by qPCR, were reduced by $>75 \%$ in PaskBKO mouse islets vs controls (Fig. 1d, left), with concomitant reduction in beta cell PASK protein content, as assessed by immunohistochemistry (Fig. 1e). No change in Ins 2 mRNA levels (Fig. 1d, right) was detected in the same islets. By contrast, differences in Pask mRNA could not be detected between PaskAKO and wild-type (WT) islets (not shown), likely reflecting the low abundance of alpha cells in rodent islets ( $20 \%$ of all cells) [35] and expected $20-50 \%$ deletion with the $P p g^{\mathrm{Cre}}$ used here [21]. Correspondingly, in PaskAKO islets, immunohistochemical analysis revealed a $42.6 \pm 17.5 \%$ overlap of the signal from anti-PASK antibody with that from glucagon antibody, whereas this value was close to $100 \%$ in WT islets (Fig. 1f).

Pancreatic PaskBKO slightly impairs glucose homeostasis after high-fat feeding Measured in male mice at 8 and 20 weeks of age, PaskBKO animals on a normal chow diet displayed normal weight gain (Fig. 2a) and normal glucose homeostasis during IPGTT compared with WT littermates (Fig. $2 \mathrm{~b}, \mathrm{c})$. Both in vivo $(1 \mathrm{~g} / \mathrm{kg}$; Fig. $3 \mathrm{a}, \mathrm{b})$ and in vitro (Fig. 3c) glucose-stimulated insulin secretion were unaffected by the deletion of Pask.

In order to provide a metabolic stress on the beta cell, where effects of Pask deletion might become apparent, we challenged PaskBKO mice with a high-fat diet for 12 weeks. Weight gain between WT and null mice was not significantly different (Fig. 2d). PaskBKO animals exposed to a high-fat diet had an elevated glucose peak during IPGTT compared with WT littermates (Fig. 2e), although no significant differences in AUC for glucose excursions (Fig. 2e) or plasma insulin (Fig. 3a, b) were observed.

\section{Pancreatic beta cell mass is lowered in PaskBKO mice and} global Pask-null mice The above observations suggested that beta cell function or mass may be affected by Pask deletion. Since a beta cell phenotype may be masked or compensated for in vivo, we further investigated this in detail by performing in vitro experiments and by quantifying islet mass. To explore the latter possibility, optical projection tomography or immunohistochemistry were undertaken and revealed a $36.5 \pm 1.2 \%$ (Fig. 4a-d) and a $38 \pm 1.93 \%$ (Fig. 4e) decrease in beta cell mass in PaskBKO and global Pask-null mice, respectively. In PaskBKO animals, this reflected both increases in the number 

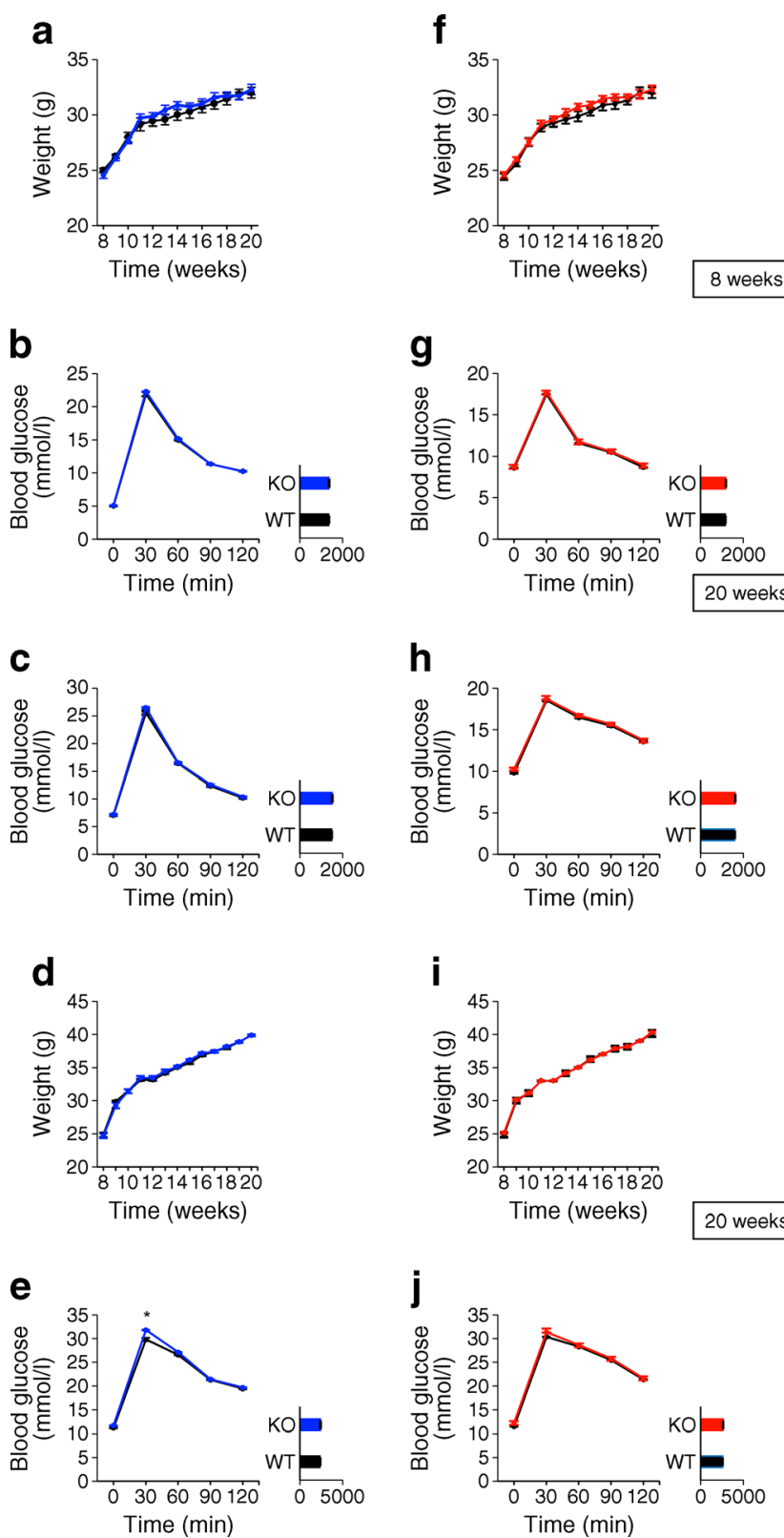

Fig. 2 PaskBKO, but not PaskAKO, mice on a high-fat diet display mild glucose intolerance after i.p. glucose injection. Body weight $(\mathbf{a}, \mathbf{f})$ and IPGTT for PaskBKO (blue), PaskAKO (red) and WT (black) male mice on a normal chow diet at $(\mathbf{b}, \mathbf{g}) 8$ weeks, and at $(\mathbf{c}, \mathbf{h}) 20$ weeks. Body weight (d, i) and IPGTT for PaskBKO (blue), PaskAKO (red) and WT (black) male mice on a high-fat diet at (e, j) 20 weeks; $n=7$ for each genotype; ${ }^{*} p<0.05$ by Student's $t$ test with Bonferroni correction; AUCs (insets) given in arbitrary units

of smaller, and decreases in the number of larger, islets (Fig. 4d). Interestingly, after maintenance on a high-fat diet, we observed a similar decrease in beta cell mass (Fig. $5 \mathrm{c}$ ), as well as a near-complete elimination of Ki-67 staining, in beta cells of PaskBKO vs WT islets (Fig. 5a, b and d), indicating impaired proliferation under these conditions. Fed plasma insulin levels were, nonetheless, unchanged in this model (Fig. 5e).
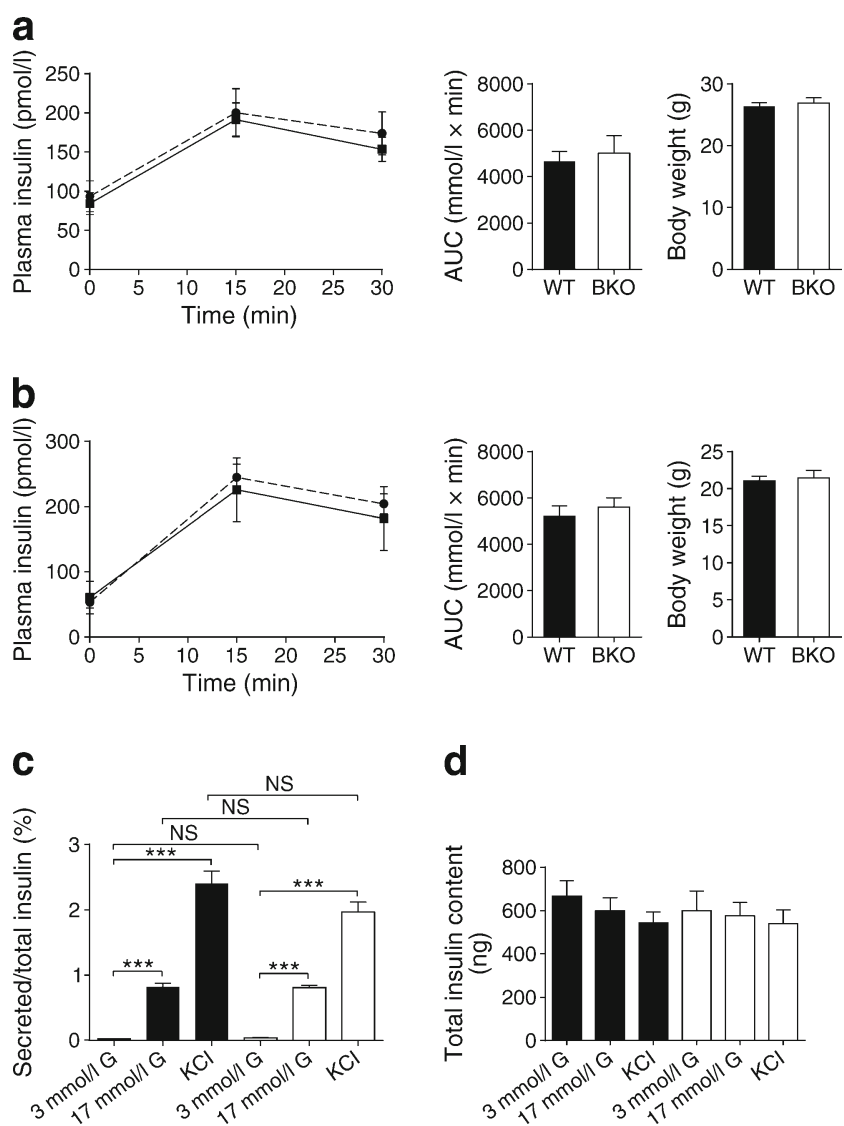

Fig. 3 PaskBKO mice display normal glucose-stimulated insulin secretion both in vivo after intraperitoneal glucose injection and in vitro from isolated islets. (a, b) In vivo glucose-stimulated insulin secretion: 13- to 15-week-old mice [PaskBKO (BKO), dashed black line on line graph or white column on histogram, male (a) $n=4$, female (b) $n=5$; WT, solid black line on line graph or black bar on histogram, male (a) $n=5$, female (b) $n=6]$ were injected with $3 \mathrm{~g}$ glucose/kg body weight. (c) In vitro glucose-stimulated insulin secretion from islets isolated from PaskBKO (white columns, $n=4$ ) and WT (black columns, $n=4$ ) mice at $3 \mathrm{mmol} / \mathrm{l}$ and $17 \mathrm{mmol} / 1$ glucose $(\mathrm{G})$ or $3 \mathrm{mmol} / 1$ glucose plus $20 \mathrm{mmol} / \mathrm{KCl}$. Statistical comparisons were made using one-way ANOVA; $* * * p<0.001$

Pancreatic alpha cell mass is lowered in global Pask-null mice but increased in PaskAKO mice Immunohistochemical analysis revealed a $47.4 \pm 0.7 \%$ decrease in alpha cell mass in global Pask-null mice (Fig. 4g). Whilst no significant changes in beta cell mass were observed in PaskAKO mice (Fig. 6a-c), a $21.9 \pm 4.6 \%$ increase in alpha cell mass was detected in PaskAKO vs WT mice (Fig. 6d).

Abnormal counterregulatory responses and glucagon secretion in PaskAKO mice Male PaskAKO mice on a normal chow diet or a high-fat diet showed no significant differences in weight gain or glucose tolerance between 8 and 20 weeks (Fig. 2f-j).

We performed hyperinsulinaemic-hypoglycaemic clamps to explore the impact of deleting Pask in the alpha cell on in vivo glucagon production. Strikingly, upon insulin infusion, the decrease in glycaemia was more rapid in PaskAKO 
Fig. 4 Beta and alpha cell volume and plasma insulin levels in PaskBKO and global Pask-null mice. Beta cell volume was measured by optical projection tomography, as described in the Methods section. Representative images of pancreases from WT (a) and PaskBKO (b) mice are shown, with insulin staining in green; scale bar, $500 \mu \mathrm{m}$. Quantification of beta cell relative to total pancreas volume $(\mathbf{c})$ and size distribution of islets (d); PaskBKO in white, WT in black. (e, g) Beta and alpha cell mass and (h) plasma insulin levels after an IPGTT in global Pask-null mice (KO, white columns or dotted line; WT in black columns or solid line) were assessed by wide-field microscopy and following immunohistochemistry, respectively. (f) Representative images of pancreatic sections from WT and global Pask-null mice; scale bar, $1000 \mu \mathrm{m}$. Data were from at least four mice per genotype, aged 20 weeks; ${ }^{*} p \leq 0.05$ by Student's $t$ test with Bonferroni correction for multiple tests
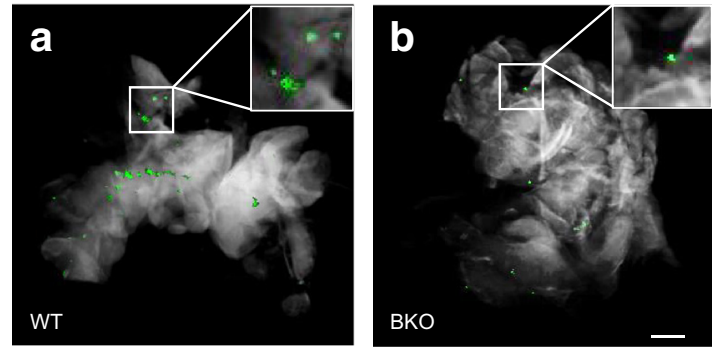

C

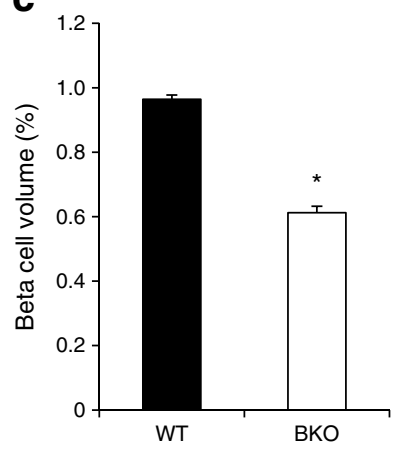

d

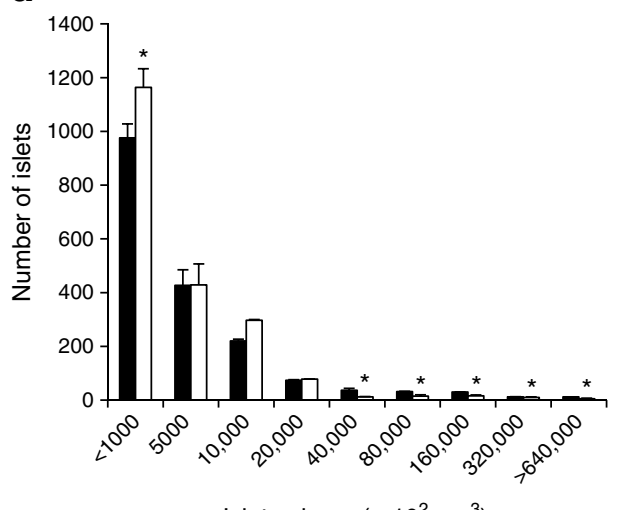

Islet volume $\left(\times 10^{2} \mu \mathrm{m}^{3}\right)$

e

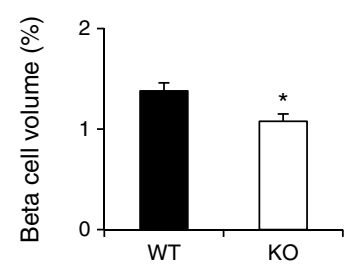

g

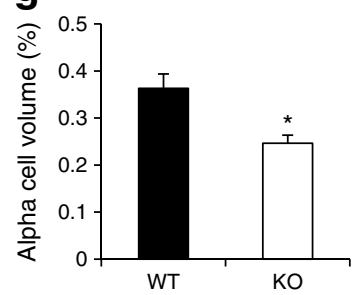

f
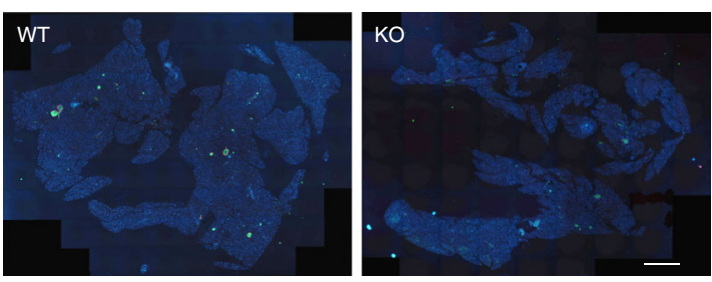

h

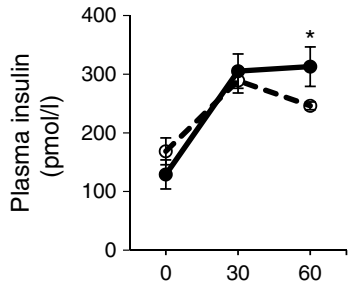

animals vs WT mice, with the former reaching $0.5 \mathrm{~g} / 1$ at around 40 min compared with $60 \mathrm{~min}$ in controls (Fig. 7a). Furthermore, during the time period $60-120 \mathrm{~h}$, when glycaemia was similar in both groups (around $0.5 \mathrm{~g} / \mathrm{l}$ ), it was necessary to infuse more glucose in PaskAKO mice, confirming a likely lack of counterregulatory response in these mice (Fig. 7b). Although a small but significant increase in glucagon levels was apparent prior to the onset of the glucose clamps (Fig. 7c), glucagon release at 120 min displayed a strong tendency towards a lowering in the knockout mice (Fig. 7c). Similarly, glucagon secretion from isolated islets was significantly reduced in response to low glucose
(Fig. 7d). By contrast, we observed no differences in insulin tolerance between mice of either genotype in vivo (Fig. 7e).

\section{Discussion}

We provide here the first description of mice deleted highly selectively for Pask in the pancreatic alpha or beta cell.

We were able to demonstrate a robust lowering of Pask expression in islets in both PaskBKO and PaskAKO mice, reflecting the expected efficiency of the Ins $1^{\text {Cre }}$ deleter strain (found to recombine in $>94 \%$ of beta cells, with no significant 

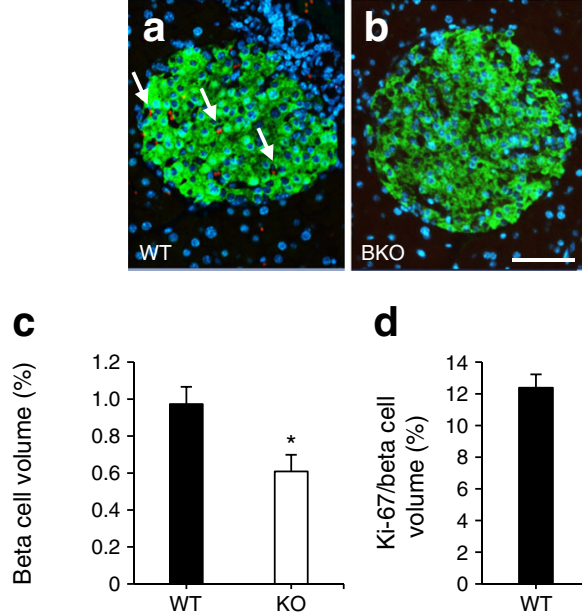

d
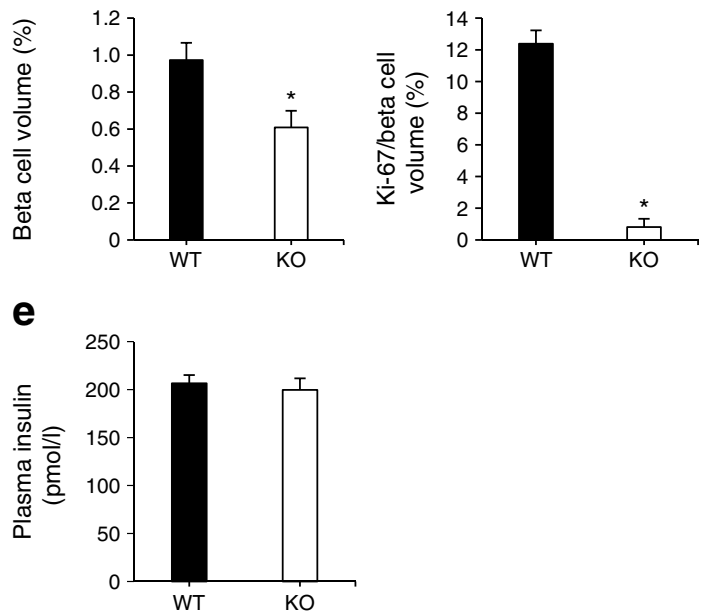

Fig. 5 PaskBKO islets from mice that have been exposed to a high-fat diet have lowered beta cell mass and Ki-67 immunoreactivity. Ki-67 levels (revealed using Alexa 568, red) and insulin (revealed using Alexa 488, green) were assessed by immunohistochemistry, as described in the Methods section. Blue indicates DAPI staining for nuclei. Representative images of islets from WT (a) and PaskBKO (b) islets and quantification of beta cell mass (c) are shown. Ki-67 expression is shown as a percentage of beta cell volume (d). Plasma insulin content is shown in (e). Arrows indicate Ki-67 staining (a); scale bar, $50 \mu \mathrm{m}$; ${ }^{*} p \leq 0.05$ by Student's $t$ test with Bonferroni correction

off-target recombination in other cell types) $[32,36]$ and the $\mathrm{Ppg}^{\mathrm{Cre}}$ strain (which recombines in $20-50 \%$ of alpha cells) [37].

Role of PASK in the beta cell Whilst insulin secretion from islets was previously shown to be defective in global Pask-null mice [27], our own studies with this model [29] indicated that the stimulation of insulin release was normal. However, Ins 2 and $P d x 1$ gene expression were both substantially lower in knockout islets, and the pancreatic content of the hormone was sharply reduced [29]. In the present study using the Ins $1^{\text {Cre }}$ line $[36,38]$ to inactivate Pask in the beta cell, we did not detect decreases in insulin mRNA measured per islet, but did observe a significant lowering of beta cell mass throughout the pancreas (Fig. 4c, d), which was paralleled by decreased Ki-67 immunoreactivity when measured after a high-fat diet (Fig. 5a-d). Furthermore, in vivo measurements of insulin revealed no significant decreases in plasma insulin following an IPGTT in PaskBKO mice when measured at 13-15 weeks (Fig. 3a, b), although there was a tendency towards lowered plasma insulin when measured
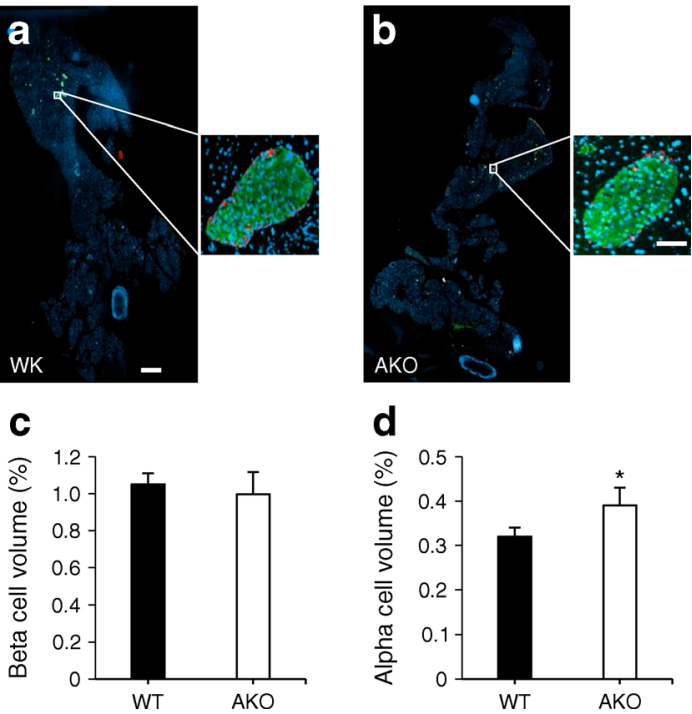

Fig. 6 PaskAKO mice display increased alpha cell mass. Immunohistochemical analysis of pancreases from male PaskAKO mice at 20 weeks of age was performed as described in the Methods section. Insulin and glucagon were revealed using Alexa 488 (green) and 568 (red), respectively, with nuclei labelled using DAPI (blue). (a, b) Typical images from pancreatic sections from WT littermate controls (black bars) and PaskAKO (white bars) mice, respectively. Scale bar, $500 \mu \mathrm{m}$; insets show enlarged images of a typical islet; inset scale bar, $50 \mu \mathrm{m}$. (c, d) Per cent beta and alpha cell volume determined using Fiji. Data are from seven mice; ${ }^{*} p<0.05$ using Student's $t$ test with Bonferroni correction

between 16 and 20 weeks (data not shown). By contrast, global Pask-null mice displayed both decreases in beta cell mass (Fig. 4e) and in vivo insulin secretion (Fig. 4h).

The reason(s) for the modest effect of beta cell-specific elimination of PASK, in contrast to findings in the global Pask-null mouse here and in earlier reports [26, 29-31], is unclear. However, these results may suggest that changes in the global Pask-null mice reflect either (1) a requirement for PASK during the development of beta cell progenitors, and/or (2) the downstream consequences of Pask deletion elsewhere in the body, possibly including the brain [39]. With respect to the first, Semache and colleagues [31] have previously reported that PASK regulates the stability of pancreatic duodenal homeobox 1, a key regulator of the insulin gene [40], which might be expected to restrict beta cell development and may underlie an observed decrease of $\sim 37 \%$ beta cell mass in the PaskBKO mouse found here. Of note, such a decrease is not expected to induce glucose dyshomeostasis, given that the destruction of $50 \%$ of beta cells is without effect in mice [41]. Thus, impaired glucose intolerance in PaskBKO mice after a high-fat diet is likely to reflect additional changes to pancreatic beta cell function and glucose sensing.

Role of PASK in the alpha cell Our previous findings [29] pointed to a role for PASK in the control of glucagon secretion. Here, we demonstrate this role in vivo after selective 
Fig. 7 Hyperinsulinaemichypoglycaemic clamps in PaskAKO and WT mice, and release of glucagon from isolated islets. Blood glucose levels (a) and glucose infusion rate (b) measured in PaskAKO (dotted line) and WT littermates (solid line) during insulin $\left(1.2 \mathrm{U} \mathrm{kg}^{-1} \mathrm{~h}^{-1}\right)$ and glucose (gluc, 20\%) co-infusion $5 \mathrm{~min}$ after bolus insulin infusion (0 min). The glucose infusion rate is indicated in (b). (c) Blood glucagon measurement before $(0 \mathrm{~min})$ and at the end $(120 \mathrm{~min})$ of the clamp. Data are expressed as means \pm SEM; $n=3-5$ mice per genotype. (d) Glucagon release from PaskAKO mouse and control islets in response to low $(0.5 \mathrm{mmol} / \mathrm{l})$ or high $(10 \mathrm{mmol} / \mathrm{l})$ glucose $(\mathrm{G})$. (e) Insulin $(0.75 \mathrm{U} / \mathrm{kg}, 0 \mathrm{~min})$ tolerance test on 24-week-old male PaskAKO $(n=3$; dotted line) or WT ( $n=5$; solid line) mice. ${ }^{*} p<0.05,{ }^{* *} p<0.01$ by Student's $t$ test with Bonferroni correction a
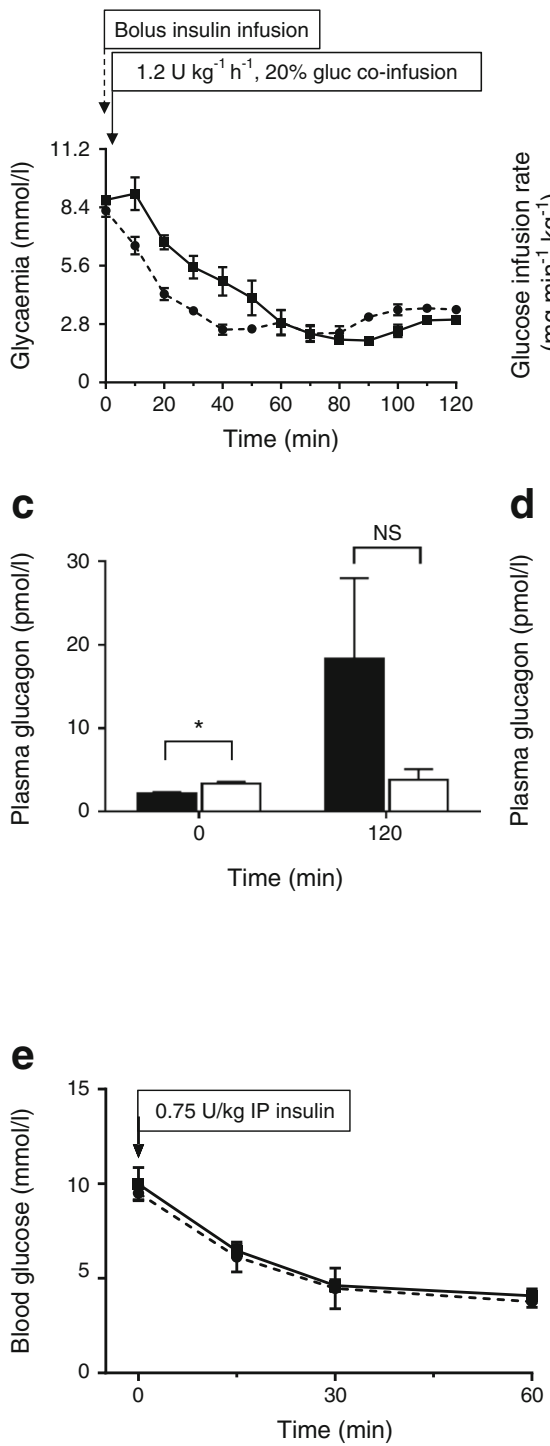

b

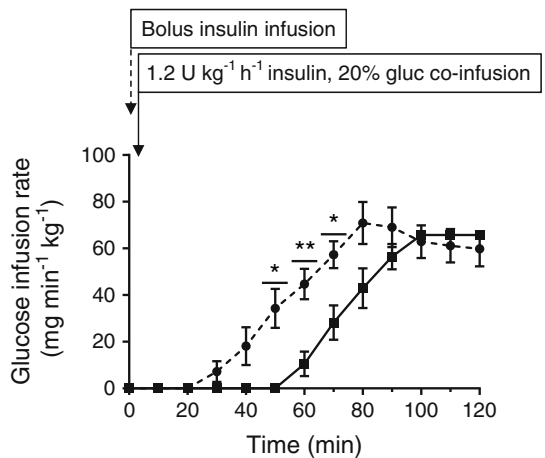

d

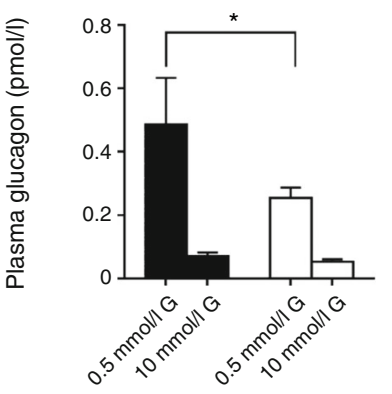

AUC $(\mathrm{mmol} / \mathrm{l} \times \mathrm{min})$
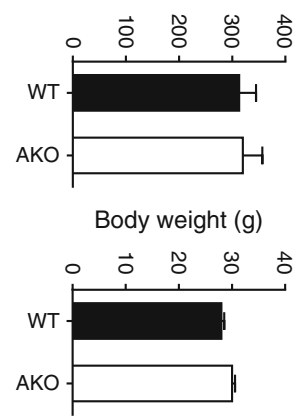

deletion from the alpha cell population. Unexpectedly, whilst we confirmed our previous finding of enhanced basal release of the hormone in global Pask-null mice (Fig. 7c), suggesting that PASK restricts glucagon release at normal levels of glycaemia, we now demonstrate that PASK is required for the stimulation of glucagon release during hypoglycaemia. Thus, glycaemia was lowered more rapidly in response to insulin infusion in PaskAKO mice than in controls, despite higher glucose infusion rates (Fig. 7). Of note, these changes were apparent despite deletion in $57.4 \%$ of alpha cells with the Cre deleter strain used here [21, 37]. Given the observed decrease in alpha cell mass of $\sim 47 \%$ in the global Pask-null mouse (Fig. 4g), we hypothesised that decreases in alpha cell number may contribute to impaired glucagon production in PaskAKO mice. However, PaskAKO mice displayed an increase in alpha cell mass (Fig. 6d), indicating that alpha cell dysfunction is likely to be the major contributor to the phenotype displayed by PaskAKO mice.

The reasons for the differences in the impact on alpha cell mass of global vs cell type-specific Pask deletion are unclear. Furthermore, the above findings are in contrast to earlier observations with global Pask-null animals [29], in which fasting glucagon levels were higher in Pask-null mice than in WT mice, and the ability of high glucose to suppress glucagon secretion was impaired in islets from the former. What may underlie these differences? Again, actions of the kinase at extrapancreatic sites (such as liver, adipocytes and brain) are one possibility, whilst a role for PASK in restricting the production of alpha cell progenitors, mooted above, may provide an alternative explanation. In support of this idea, alpha cell mass was decreased in global Pask-null mice (Fig. 4g). Alpha cell function is 
also regulated through insulin secretion by neighbouring beta cells and it is therefore conceivable that the effects on glucagon secretion observed in the whole-body knockout mice may reflect a combination of defects in all pancreatic cell types in this model that are not replicated in the present cell type-specific models. It is currently unknown, for example, whether PASK has any function in pancreatic delta cells, although somatostatin is a regulator of both alpha and beta cell function [42]. Nevertheless, the present results demonstrate a clear and cell-autonomous role for PASK in the acute control of glucagon secretion (Fig. 7c, d) and beta cell mass (Fig. 5). The precise molecular mechanisms through which the kinase acts will need further exploration, and possibly the generation of mice bearing selectively-labelled alpha cells to permit studies at the single cell level [37].

Conclusions The present findings indicate that PASK plays critical roles in the biology of both pancreatic beta and alpha cells in vivo. However, we reveal striking differences between the glycaemic phenotype of animals deleted globally for the kinase vs the cell type-specific knockouts, demonstrating likely important roles for tissue interactions or actions at early developmental stages.

Acknowledgements We thank M.-S. Nguyen-Tu for help with OGTTs, and A. Martinez-Sanchez for proofreading the manuscript.

Funding The study was funded by grants to GAR from Diabetes UK (Project BDA 11/0004210), the Wellcome Trust (Programme 081958/Z/07/Z; Senior Investigator Award WT098424AIA), and the MRC (UK; Project GO401641; Programme MR/J0003042/1). GdSX thanks Diabetes UK (BDA 13/0004672) and the European Foundation for the Study of Diabetes-Merck Sharp and Dohme for project grants. The work leading to this publication also received support from the Innovative Medicines Initiative Joint Undertaking under grant agreement no. 155005 (IMIDIA) (GAR, CM, PLH), resources of which are composed of financial contributions from the European Union's Seventh Framework Programme (FP7/20072013) and the European Federation of Pharmaceutical Industries and Associations.

Duality of interest KM-B, AP-K, SB, AR and AM are employees of AstraZeneca. The remaining co-authors declare that there is no duality of interest associated with their involvement in this manuscript.

Contribution statement GAR and GdSX conceived the project and co-wrote the article with FS. KM-B, AP-K, SB, AR and AM generated the floxed mouse line. PLH provided the $P p g^{\mathrm{Cre}}$ mice and co-designed the strategy to generate and genotype the PaskAKO transgenic line. FS, AM, HS and GdSX generated and genotyped the conditional knockout mice, and performed IPGTTs, gene expression analysis, and insulin and glucagon secretion assays. JMG, PF, BM, RY, AM and GdSX performed immunohistochemical and optical projection tomography analysis. CM, SM-L, and EP performed hypoglycaemic clamps, and JL and JG the glucagon assays. All authors were involved in drafting the article or revising it critically, and all authors gave final approval of the version to be published. GAR is the guarantor of this work.
Open Access This article is distributed under the terms of the Creative Commons Attribution 4.0 International License (http:// creativecommons.org/licenses/by/4.0/), which permits unrestricted use, distribution, and reproduction in any medium, provided you give appropriate credit to the original author(s) and the source, provide a link to the Creative Commons license, and indicate if changes were made.

\section{References}

1. Guariguata L, Whiting DR, Hambleton I, Beagley J, Linnenkamp U, Shaw JE (2014) Global estimates of diabetes prevalence for 2013 and projections for 2035. Diabetes Res Clin Pract 103:137149

2. Kahn SE, Zraika S, Utzschneider KM, Hull RL (2009) The beta cell lesion in type 2 diabetes: there has to be a primary functional abnormality. Diabetologia 52:1003-1012

3. Parker VE, Savage DB, O'Rahilly S, Semple RK (2011) Mechanistic insights into insulin resistance in the genetic era. Diabet Med 28:1476-1486

4. Thorens B, Sarkar HK, Kaback HR, Lodish HF (1988) Cloning and functional expression in bacteria of a novel glucose transporter present in liver intestine kidney and B-pancreatic islet cells. Cell 55:281-290

5. Rutter GA, Pullen TJ, Hodson DJ, Martinez-Sanchez A (2015) Pancreatic beta cell identity, glucose sensing and the control of insulin secretion. Biochem J 466:202-218

6. Hutton JC, Sener A, Herchuelz A et al (1980) Similarities in the stimulus-secretion coupling mechanisms of glucose- and 2-keto acid-induced insulin release. Endocrinology 106:203-219

7. Tarasov AI, Griffiths EJ, Rutter GA (2012) Regulation of ATP production by mitochondrial $\mathrm{Ca}^{2+}$. Cell Calcium 52:28-35

8. Ashcroft FM, Rorsman P (2013) $\mathrm{K}_{\text {ATP }}$ channels and islet hormone secretion: new insights and controversies. Nat Rev Endocrinol 9: 660-669

9. Rutter GA, Theler J-M, Murta M, Wollheim CB, Pozzan T, Rizzuto $\mathrm{R}$ (1993) Stimulated $\mathrm{Ca}^{2+}$ influx raises mitochondrial free $\mathrm{Ca}^{2+}$ to supramicromolar levels in a pancreatic $\beta$-cell line: possible role in glucose and agonist-induced insulin secretion. J Biol Chem 268: 22385-22390

10. Rutter GA, Pralong W-F, Wollheim CB (1992) Regulation of mitochondrial glycerol phosphate dehydrogenase by $\mathrm{Ca}^{2+}$ within electropermeabilized insulin secreting cells (INS-1). Biochim Biophys Acta 1175:107-113

11. Rutter GA (2004) Visualising insulin secretion. The Minkowski lecture 2004. Diabetologia 47:1861-1872

12. Henquin JC (2009) Regulation of insulin secretion: a matter of phase control and amplitude modulation. Diabetologia 52:739-751

13. Tarussio D, Metref S, Seyer P et al (2014) Nervous glucose sensing regulates postnatal beta cell proliferation and glucose homeostasis. J Clin Investig 124:413-424

14. Ravier MA, Rutter GA (2005) Glucose or insulin, but not zinc ions, inhibit glucagon secretion from mouse pancreatic $\alpha$-cells. Diabetes 54:1789-1797

15. Gopel SO, Kanno T, Barg S, Weng X, Gromada J, Rorsman P (2000) Regulation of glucagon release in mouse-cells by KATP channels and inactivation of TTX-sensitive $\mathrm{Na}+$ channels. J Physiol 528:509-520

16. Liu YJ, Vieira E, Gylfe E (2004) A store-operated mechanism determines the activity of the electrically excitable glucagon-secreting pancreatic alpha-cell. Cell Calcium 35:357-365

17. Rutter GA, Leclerc I (2009) The AMP-regulated kinase family: enigmatic targets for diabetes therapy. Mol Cell Endocrinol 297: 41-49 
18. da Silva Xavier G, Leclerc I, Varadi A, Tsuboi T, Moule SK, Rutter GA (2003) Role for AMP-activated protein kinase in glucosestimulated insulin secretion and preproinsulin gene expression. Biochem J 371:761-774

19. da Silva Xavier G, Leclerc I, Salt IP et al (2000) Role of AMPactivated protein kinase in the regulation by glucose of islet betacell gene expression. Proc Natl Acad Sci U S A 97:4023-4028

20. Kuznetsov JN, Leclerc GJ, Leclerc GM, Barredo JC (2011) AMPK and Akt determine apoptotic cell death following perturbations of one-carbon metabolism by regulating ER stress in acute lymphoblastic leukemia. Mol Cancer Ther 10:437-447

21. Sun G, da Silva Xavier G, Gorman T et al (2015) LKB1 and AMPKalpha1 are required in pancreatic alpha cells for the normal regulation of glucagon secretion and responses to hypoglycemia. Mol Metab 4:277-286

22. Hofer T, Spielmann P, Stengel P et al (2001) Mammalian PASKIN, a PAS-serine/threonine kinase related to bacterial oxygen sensors. Biochem Biophys Res Commun 288:757-764

23. Rutter J, Michnoff CH, Harper SM, Gardner KH, McKnight SL (2001) PAS kinase: an evolutionarily conserved PAS domainregulated serine/threonine kinase. Proc Natl Acad Sci U S A 98: 8991-8996

24. Sabatini PV, Lynn FC (2015) All-encomPASsing regulation of betacells: PAS domain proteins in beta-cell dysfunction and diabetes. Trends Endocrinol Metab 26:49-57

25. Grose JH, Smith TL, Sabic H, Rutter J (2007) Yeast PAS kinase coordinates glucose partitioning in response to metabolic and cell integrity signaling. EMBO J 26:4824-4830

26. da Silva Xavier G, Rutter J, Rutter GA (2004) Involvement of PerArnt-Sim (PAS) kinase in the stimulation of preproinsulin and pancreatic duodenum homeobox 1 gene expression by glucose. Proc Natl Acad Sci U S A 101:8319-8324

27. Hao HX, Cardon CM, Swiatek W et al (2007) PAS kinase is required for normal cellular energy balance. Proc Natl Acad Sci U S A 104:15466-15471

28. Semplici F, Vaxillaire M, Fogarty S et al (2011) A human mutation within the per-ARNT-sim (PAS) domain-containing protein kinase (PASK) causes basal insulin hypersecretion. J Biol Chem 286: 44005-44014

29. da Silva Xavier G, Farhan H, Kim H et al (2011) Per-arnt-sim (PAS) domain-containing protein kinase is downregulated in human islets in type 2 diabetes and regulates glucagon secretion. Diabetologia 54:819-827

30. Fontes G, Semache M, Hagman DK et al (2009) Involvement of Per-Arnt-Sim kinase and extracellular-regulated kinases-1/2 in palmitate inhibition of insulin gene expression in pancreatic betacells. Diabetes 58:2048-2058

31. Semache M, Zarrouki B, Fontes G et al (2013) Per-Arnt-Sim kinase regulates pancreatic duodenal homeobox-1 protein stability via phosphorylation of glycogen synthase kinase 3 beta in pancreatic beta-cells. J Biol Chem 288:24825-24833

32. Thorens B, Tarussio D, Maestro MA, Rovira M, Heikkila E, Ferrer $\mathrm{J}$ (2015) Ins1 knock-in mice for beta cell-specific gene recombination. Diabetologia 58:558-656

33. Herrera PL (2000) Adult insulin- and glucagon-producing cells differentiate from two independent cell lineages. Development 127:2317-2322

34. Katschinski DM, Marti HH, Wagner KF et al (2003) Targeted disruption of the mouse PAS domain serine/threonine kinase PASKIN. Mol Cell Biol 23:6780-6789

35. Elayat AA, el-Naggar MM, Tahir M (1995) An immunocytochemical and morphometric study of the rat pancreatic islets. J Anat 186: 629-637

36. Kone M, Pullen TJ, Sun G et al (2014) LKB1 and AMPK differentially regulate pancreatic beta-cell identity. FASEB J 28:49724985

37. Solomou A, Meur G, Bellomo E et al (2015) The zinc transporter Slc30a8/ZnT8 is required in a subpopulation of pancreatic $\alpha$-cells for hypoglycemia-induced glucagon secretion. J Biol Chem 290: 21432-21442

38. Patel D, Ythier D, Brozzi F, Eizirik DL, Thorens B (2015) Clic4, a novel protein that sensitizes $\beta$-cells to apoptosis. Mol Metab 4:253264

39. Hurtado-Carneiro V, Roncero I, Egger SS et al (2014) PAS kinase is a nutrient and energy sensor in hypothalamic areas required for the normal function of AMPK and mTOR/S6K1. Mol Neurobiol 50: 314-326

40. Melloul D, Marshak S, Cerasi E (2002) Regulation of insulin gene transcription. Diabetologia 45:309-326

41. Vinet L, Lamprianou S, Babic A et al (2015) Targeting GLP-1 receptors for repeated magnetic resonance imaging differentiates graded losses of pancreatic beta cells in mice. Diabetologia 58: 304-312

42. Hauge-Evans AC, King AJ, Carmignac D et al (2009) Somatostatin secreted by islet delta-cells fulfills multiple roles as a paracrine regulator of islet function. Diabetes 58:403-411

43. Kikani CK, Antonysamy SA, Bonanno JB et al (2010) Structural bases of PAS domain-regulated kinase (PASK) activation in the absence of activation loop phosphorylation. J Biol Chem 285: 41034-41043 